\title{
Blood pressure changes after intravitreal bevacizumab in patients grouped by ocular pathology
}

Eye (2011) 25, 1669; doi:10.1038/eye.2011.266

Correction to: Eye (2010) 24, 1320-1324; doi:10.1038/ eye.2010.22; published online 9 April 2010

Since the publication of this paper, the authors have noted that $\mathrm{Y}-\mathrm{R}$ Chung was incorrectly listed as the sole first author. Instead, Y-R Chung and K Lee are the co-first authors as listed above.
Y-R Chung ${ }^{1,3}, \mathrm{~K} \mathrm{Lee}^{2,3}, \mathrm{E}-\mathrm{H}$ Cho' ${ }^{1}$ and HM Lew ${ }^{1}$

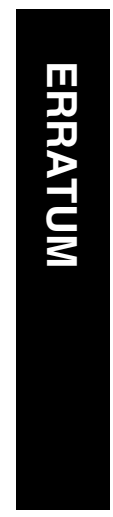

The typesetters would like to apologise for this error.

Department of Ophthalmology, Ajou University School of Medicine, Suwon, Republic of Korea

2Department of Ophthalmology, Gunsan Medical Center, Gunsan, Jeollabuk-Do, Republic of Korea

${ }^{3}$ Co-first authors 